\title{
Encontro de larvas de Anopheles (Nyssorhynchus) argyritarsis em criadouros artificiais no Estado de Mato Grosso
}

\author{
Anopheles (Nyssorbynchus) argyritarsis larvae found in artificial \\ breeding sites in the State of Mato Grosso
}

\author{
Júlia dos Santos Silva ${ }^{1}$, Anagela Maria Acel², Anthony Érico Guimarães ${ }^{1}$ \\ e Jeronimo Alencar ${ }^{1}$
}

\begin{abstract}
RESUMO
Cita-se o encontro de formas imaturas de culicídeos em caixas d'água colocadas no nível do solo. Foram encontradas larvas de Anopheles argyritarsis e Aedes albopictus no município de Várzea Grande (MT). Considera-se que estes recipientes artificiais possam constituir locais adicionais utilizáveis como criadouros de anofelinos.
\end{abstract}

Palavras-chaves: Anopheles. Ecologia de vetores. Recipientes artificiais. Larva.

\begin{abstract}
The finding of immature forms of culicid mosquitoes in water tanks at ground level is reported. Anopheles argyritarsis and Aedes albopictus were found in the municipality of Várzea Grande (MT). It is considered that these artificial containers may constitute additional locations that anophelines can use as breeding sites.
\end{abstract}

Key-words: Anopheles. Vector ecology. Artificial containers. Larva.

0 encontro de formas imaturas de Anopheles Meigen, 1818 criando-se em recipientes artificiais fora do ambiente de laboratório é considerado anômalo, e por isso apresenta grande importância epidemiológica ${ }^{67}$. Anopheles argyritarsis Robineau-Desvoidy, 1987 é um mosquito cujos locais de criação das formas imaturas são muito variados, dentro da gama de coleções líquidas terrestres, como margens de rios, lagos naturais ou artificiais ${ }^{5}$. Esta espécie tem ampla distribuição, usualmente ocorrendo em pequenas proporções ${ }^{910}$. No entanto, considerando que Anopheles marajoara Galvão \& Damasceno, 1942 tem se tornado vetor principal da malária em uma região no nordeste da Amazônia a partir de modificações ambientais ${ }^{2}$, as espécies ditas secundárias também devem ser observadas com cuidado.

Embora Forattini ${ }^{5}$ relate que a capacidade vetora de Anopheles argyritarsis tem sido objeto de opiniões contraditórias, fato atribuído a possíveis identificações equivocadas, resultantes de confusão com representantes de outras espécies, que posteriormente foram reconhecidas como vetores, não devemos desconsiderar que estudos experimentais realizados em laboratório demonstraram que a espécie apresenta capacidade de transmitir o Plasmodium vivax ${ }^{4}$.
Durante a execução do Programa de Controle da Febre Amarela e Dengue da Secretaria Municipal de Saúde de Várzea Grande, Estado do Mato Grosso, foram realizadas coletas de imaturos nos anos de 2005 e 2006.

0 município de Várzea Grande, com extensão territorial de 949,53 $\mathrm{km}^{2}$, está localizado na micro-região de Cuiabá no Estado de Mato Grosso, fazendo limites com as Cidades de Cuiabá, Acorizal, Jangada, Santo Antônio do Leverger e Nossa Senhora do Livramento. Situada no relevo Baixada do Rio Paraguai e calha do Rio Cuiabá, a $185 \mathrm{~m}$ de altitude, Várzea Grande pertence à Baixada Cuiabana ou Periplanície Cuiabana, pelas coordenadas: 1532'30" S 56²17'18" W. Apresenta clima tropical continental tipo quente subúmido, solo predominantemente de argila avermelhada, principalmente na faixa marginal do Rio Cuiabá e vegetação composta por savana arbórea aberta (cerrado), capoeira e mata ciliar. Segundo o Censo de 2000 do Instituto Brasileiro de Geografia e Estatísticas (IBGE), o município possui população de 248.728 mil habitantes, dos quais 210.849 mil morando em área urbana.

0 monitoramento entomológico no município de Várzea Grande teve como objetivo caracterizar a situação vetorial

\footnotetext{
1. Laboratório de Diptera, Instituto Oswaldo Cruz, Fundação Oswaldo Cruz, Rio de Janeiro, RJ. 2. Centro de Controle de Zoonoses de Várzea Grande, Secretaria Municipal de Saúde, Cuiabá, MT.

Endereço para correspondência: MSc Júlia dos Santos Silva. Laboratório de Diptera/FIOCRUZ. Av. Brasil 4365, Manguinhos, $21045-900$ Rio de Janeiro, RJ.

Tel: 5521 2562-1495

e-mail: juliass@ioc.fiocruz.br

Recebido para publicação em: 30/07/2007

Aceito em: 02/06/2008
} 
desse município no controle da febre amarela e dengue. No entanto, foram coletadas em área urbana 56 larvas de Anopheles argyritarsis, em recipientes artificiais do tipo caixa d'água localizada no nível do solo. As caixas d'água analisadas tinham 5001 de capacidade, tendo sido coletadas em média oito larvas em cada depósito positivo (Figura 1).

Junto com as larvas de Anopheles argyritarsis, foram encontradas também três larvas de Aedes albopictus Skuse, 1894. 0 mapa de distribuição de Aedes albopictus, não inclui o município de Várzea Grande como positivo ${ }^{11}$. Assim, este trabalho demonstra a ampliação da área de distribuição da espécie no Estado.

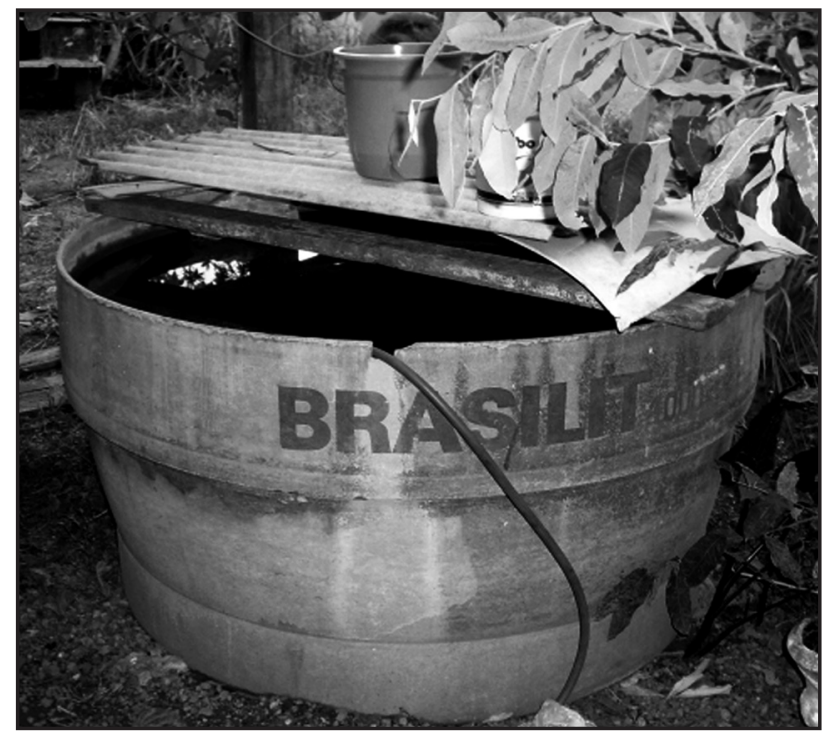

Figura 1 - Aspecto do recipiente artificial do tipo caixa d'água, no qual foram encontradas formas imaturas de Anopheles argyritarsis $e$ Aedes albopictus.

Os espécimes foram encaminhados ao Laboratório de Diptera do Instituto Oswaldo Cruz, da Fundação Oswaldo Cruz, RJ, para a determinação específica. A identificação dos espécimes foi realizada pela observação direta dos caracteres morfológicos evidenciáveis ao microscópio de luz transmitida, utilizando chaves dicotômicas ${ }^{35}$.

Horsfall $^{8}$ relatou que Anopheles argyritarsis apresenta preferência por coleções líquidas no solo, normalmente com vegetação emergente, e dotadas de certa movimentação, restringindo o encontro dessa espécie em criadouros artificiais, localizado acima do solo e ao abrigo da incidência das excessivas elevações da temperatura ambiente. Dessa maneira, o encontro de Anopheles argyritarsis em um recipiente artificial, sem movimentação líquida, desprovido de vegetação emergente e em companhia de Aedes albopictus, não condiz com as características dos criadouros normalmente utilizados por essa espécie.

No entanto, os resultados deste trabalho mostram-se de acordo com as observações realizadas anteriormente em São Paulo e Rio de Janeiro. Forattini cols ${ }^{6}$, observando o comportamento de culicídeos potencialmente vetores de infecções, encontraram em Pindamonhangaba duas larvas de Anopheles argyritarsis, juntamente com imaturos de Aedes albopictus e Culex quinquefasciatus Say, 1823, também em recipiente artificial do tipo caixa d'água. Carreira-Alves ${ }^{1}$, em pesquisa larvária no município de Maricá, encontrou 13 larvas de Anopheles argyritarsis em uma caixa d'água desativada e destampada.

Diante das observações realizadas, deve-se considerar que esta adaptação a tais recipientes pode ampliar os locais utilizáveis como criadouros de alguns anofelinos, e que se torna recomendável o monitoramento nos bairros positivos.

\section{REFERÊNCIAS}

1. Carreira-AlvesJR. Encontro de anofelinos do subgênero Nyssorhynchus em recipientes artificiais, Maricá, RJ, Brasil. Revista de Saúde Pública 35: 407-408, 2001.

2. Conn JE, Wilkerson RC, Segura MN, Rouza RTL, Schlichting CD, Wirtz RA, Póvoa MM. Emergence of a new Neotropical malaria vector facilitated by human migration and changes in land use. The American Journal of Tropical Medicine and Hygiene 66: 18-22, 2002.

3. Consoli RAGB, Lourenço-de-Oliveira R. Principais mosquitos de importância médica no Brasil. Fundação Oswaldo Cruz, Rio de Janeiro, 1994.

4. Faran ME, Linthicum KJ. A handbook of the Amazonian species of Anopheles (Nyssorhynchus) (Diptera: Culicidae). Mosquito Systematics 13: 1-81, 1981.

5. Forattini OP. Culicidologia Médica. Editora da Universidade de São Paulo, São Paulo, Volume 2, 2002.

6. Forattini OP, Kakitani I, Marques GRAM, Brito M. Formas imaturas de anofelíneos em recipientes artificiais. Revista de Saúde Pública 32: 189-191, 1998.

7. Forattini OP, Kakitani I, Marques GRAM, Brito M. Novos encontros de anofelíneos em recipientes artificiais. Revista de Saúde Pública 32: 598-599, 1998.

8. Horsfall WR. Mosquitoes: their bionomics and relation to disease. Hafner Publishing, New York, 1972.

9. Juri MJD, Zaidenberg M, Almirón W. Spatial distribution of Anopheles pseudopunctipennis in the Yungas de Salta rainforest, Argentina. Revista de Saúde Pública 39: 565-570, 2005.

10. Rebêlo JMM, Silva AR, Ferreira LA, Vieira JA.Anopheles (Culicidae, Anophelinae) e a malária em Buriticupu-Santa Luzia, Pré-Amazônia Maranhense. Revista da Sociedade Brasileira de Medicina Tropical 30: 107-111, 1997.

11. Santos RLC. Atualização da distribuição de Aedes albopictus no Brasil (1997-2002). Revista de Saúde Pública 37: 671-673, 2003. 